\title{
Experimentelle Heilmittel und die Ethik des Zufalls
}

\author{
Die Ebola-Epidemie wütet in Westafrika und erschüttert die Welt. Medikamente be- \\ finden sich in klinischen Tests. Die Freigabe noch in Erprobung befindlicher Medika- \\ mente wirft ethische Fragen auf, ebenso wie die Zuteilung zu Studiengruppen. \\ «Compassionate use» scheint keine Lösung.
}

Eduard Kaeser

\section{«Es ist unethisch, den Zufall nicht walten zu lassen, um zu erkennen, ob das Medikament anschlägt.»}

\section{Traitement expérimental et éthique du hasard}

Dans l'ombre médiatique de l'épidémie d'Ebola en Afrique de l'Ouest, un problème d'ordre éthique gagne en importance et en contours: une maladie mortelle et contagieuse menace la population de toute une région du monde, et nous ne disposons d'aucun remède efficace et fiable. Plusieurs médicaments se trouvent en phase de test clinique. Devrions-nous les administrer aux malades, même si leurs effets ne sont pas encore connus? Un médicament non éprouvé n'est-il pas mieux qu'aucun médicament? Une situation qui illustre parfaitement le rôle éthique central qu'exerce le hasard, déjà lors des procédures de test.

Über die Wirkungsweise von ZMapp ist man im Ungewissen. Die Amerikanerin Nancy Writebol und der Amerikaner Kent Brantly hätten ebenso gut aus anderen Gründen überleben können, etwa aufgrund ihres robusten Immunsystems, einer besseren Spitalbetreuung oder - wie Frau Writebol nicht zu betonen unterliess - eines starken Glaubens. Ein wichtiger Einwand gegen unerprobte Arzneimittel zielt auf ihre schädlichen Nebenwirkungen ab. Es handelt sich um eine heikle Frage der Balance: Was ist schädlicher, die Krankheit oder das Mittel gegen sie? Im Fall von Ebola dürfte das Mittel kaum gefährlicher sein als die Krankheit. Entscheidend ist die Frage, ob es behandelten Patienten besser, schlechter oder gleich gut geht wie nichtbehandelten. Und um dies festzustellen, braucht man Kontrollgruppen für den sogenannten Doppelblindtest. Eine Gruppe nimmt das Verum zu sich, die andere ein Placebo. Darüber, wer was einnimmt, entscheidet der Zufall. Verständlicherweise finden es viele Leute kaltherzig, wenn man Patienten ein potentiell lebensrettendes Medikament vorenthält. Aber genau das ist der sprin-
Dr. phil. nat. Eduard Kaeser

Liebeggweg 6

CH-3006 Bern tionalen Notfall der öffentlichen Gesundheit. Die Freigabe der Arzneimittel wurde an massive ethische der Behandlung, informierte Einwilligung des Patienten, Wahlfreiheit, Vertraulichkeit, Achtung de , Bewahrung ihrer Würde und Einbezug hang suft». Man spricht in diesem Zusan Deutsch etwa: Freigabe aus Barmherzigkeit. Das klingt sehr ethisch und humanitär. Aber damit verman ein Problem, quasi ein Dritte-W nicht, dass man sie als Probanden für potenti (nedikamente missbraucht? Die nerin und ein Amerikaner. Ihr Zustand verbesserte sich. Heisst das nun aber nicht, dass man sie gegenüber den Afrikanern privilegiert? Die Afrikaner kommen so oder so schlecht weg, ob man ihnen nun das Medikament gibt oder nicht: Im einen Fall nutzt man sie aus, im anderen benachteiligt man sie. 
gende Punkt. Wir haben im Fall von Ebola gar keine andere Wahl, als das Medikament vorzuenthalten. Es gibt schlicht nicht genug davon. Die Knappheit macht den zufälligen zum gerechtesten Weg. Und genau diesen Weg beschreitet der Doppelblindtest.

Es ist unethisch, den Zufall nicht walten zu lassen, um zu erkennen, ob das Medikament anschlägt. Diese These hat jüngst die Biologin Joanna Masel von der University of Arizona vertreten. Angenommen, wir haben bloss zwei Dosen des Medikaments. «Die US-Behörden hätten eine Münze werfen müssen,» so Masel, «um zu entscheiden, welcher der beiden amerikanischen Patienten ZMapp und welcher ein Placebo erhält. Und dann hätte man die andere Dosis zwei afrikanischen Patienten unter Zufallsbedingungen geben müssen. Das wäre ethisch gewesen.»

Nun kann man zwei Probandenpaare nicht gerade als aussagekräftige statistische Basis betrachten. Aber zufallsgesteuerte Tests brauchen gar keine riesigen Kontrollgruppen, um signifikante Aussagen über Medikamente zu erlauben. Der Zufall erweist sich generell als ein wichtiges Prüfmittel. Das dokumentiert ein Präzedenzfall aus dem Jahre 1948. Tuberkulose wies damals ähnliche Todesraten wie Ebola auf. Ein potentielles Mittel dagegen - Streptomycin - war knapp. Genau aus dieser Notlage heraus betrachtete man es als ethisch vertretbar, die Lotterie entscheiden zu lassen, wer das Verum und wer das Placebo erhielt. Ein Pionier der medizinischen Statistik, Bradford Hill, entwarf den ersten randomisierten Test des Streptomycins. Das war in Medizinerkreisen eher ungewöhnlich. Der Zufall war ethisch anrüchig, wissenschaftlich unseriös. Hill vermied in seinem Lehrbuch über medizinische Statistik den Begriff «randomisiert», weil er glaubte, die Ärzte würden durch ihn abgeschreckt. Nichtsdestoweniger gilt der Test des Streptomycins als die Geburtsstunde der modernen Doppelblindstudien, des Goldstandards klinischer Prüfung.

«Compassionate use» zum Trotz: Mitleid und Barmherzigkeit sind die natürlichen Feinde der Randomisierung, und damit einer Ethik des Zufalls. Der Arzt selber, sagt man, sei ein Placebo, in dem Sinne, dass er dem Patienten «gefällt» oder der Patient ihm «gefällt». Voreingenommenheiten sind menschlich, aber gerade das Menschliche kann sich - pointiert gesagt - als unethisch entpuppen. Die Verlockung des Mogelns ist allgegenwärtig. Damit ist selbstverständlich nicht der Wert einer persönlichen ArztPatient-Beziehung angezweifelt. Wie nützlich sich Placebos indes auch erweisen mögen, randomisierte Tests kontrollieren ihre «gefallende» Wirkung und hindern so «wohlmeinende» Mediziner daran, die Bereitstellung des Medikaments zu manipulieren.

Es bedarf vieler Massnahmen zur Bekämpfung der Epidemie, etwa Prophylaxe, Aufstockung des Personals und der klinischen Materials, Intensivierung der Erforschung neuer Medikamente, Produktionssteigerung - vor allem aber auch der Lotterie in der experimentellen Anwendung: eine intelligente Mixtur aus Plan und Zufall. Jede Behandlung sollte hier in codierter «Zufallsverpackung» ausgegeben werden. Niemand weiss, was darin steckt, Verum oder Placebo. Noch einmal Joanna Masel: «Eine Bedingung, ein Behandlungspaket zu erhalten, lautet, dass zurückgemeldet wird, ob der Empfänger des Pakets lebt oder gestorben ist. Das ist nicht kompliziert. Moderne klinische Tests sind in der Regel komplizierter, aber Subtilitäten spielen bei einer Krankheit wie Ebola, wo es um Leben oder Tod geht, keine grosse Rolle.»

Eine Rolle in der Zufallsabgabe von Medikamenten spielt allerdings das medizinethische Gebot der «informierten Einwilligung». Und hier öffnet sich fast unweigerlich eine Kulturkluft. Afrikanische Patienten, hört man, seien misstrauisch gegenüber der westlichen Medizin und ihren Vertretern. Wohl nicht ohne Grund. Denn dass man Probanden für riskante medizinische Versuche aus dem Pool von Menschen afrikanischen Ursprungs rekrutierte, ist in der jüngeren Medizingeschichte notorisch, von den Experimenten an schwarzen Syphilispatienten in Tuskegee, Alabama, bis zu den Versuchsimpfungen des Pharma-Riesen Pfizer an Meningitis-kranken Kindern in Nigeria. Verwundert es da, dass Afrikaner zum Medizinmann rennen und traditionelle Heilrituale praktizieren, statt sich von «fremden» Ärzten informieren zu lassen?

Hier kippt das ethische Problem in ein interkulturelles. Unter den genannten Massnahmen der Epidemiebekämpfung appelliert immer die vielleicht grundlegendste: Die Überwindung kultureller Barrieren. Wie schwierig sie ist, bekunden all jene, die im Dienst der öffentlichen Gesundheit in der Dritten Welt arbeiten. Die Sozialwissenschaftlerin Laura Seay schreibt kürzlich: «Die meisten Forscher in Entwicklungsländern - ich eingeschlossen - erzählen ähnliche Geschichten von Probanden, die nicht verstehen, warum sie ein Formular zur informierten Einwilligung unterschreiben sollen. In ihren Augen bedeutet die Bereitschaft, mit mir zu reden, Einwilligung. Ein Dokument zu unterschreiben hinterlässt zudem eine schriftliche Spur, die, so fürchten viele, von politischen Autoritäten, ausländischen Spionen oder anderen ihnen nicht Gutgesinnten gegen sie verwendet werden könnte.» Das trifft natürlich gerade auf eine von politischem und sozialem Chaos gebeutelte Gegend wie Westafrika zu.

Es ist zu erwarten, dass ZMapp und TKM in grösseren Mengen produziert und wahrscheinlich bald in randomisierten Tests geprüft werden. Wichtig wäre, dass der Zufall in Feldversuchen die kulturellen Grenzen aufhöbe. Niemand sollte mehr als eine 50-prozentige Chance haben, das Verum statt das Placebo zu erhalten. Im besten Fall hilft eine solche Lotterie, ein Mittel gegen Ebola zu finden, von dem wir überzeugt sein können, dass es wirkt. Besser wären mehrere Mittel. Dann könnten wir sie in Tests gegeneinander antreten lassen, statt gegen ein Placebo. Und der Zufall zöge sich etwas in den Hintergrund zurück. Hier wartet er allerdings, bis er das nächste Mal gerufen wird. Die Zukunft ist ihm sicher. 